\title{
U-Penn Psych hosting the package 'bayesvl'
}

The Comprehensive R Archive Network (CRAN)

Access date:

February 15, 2021
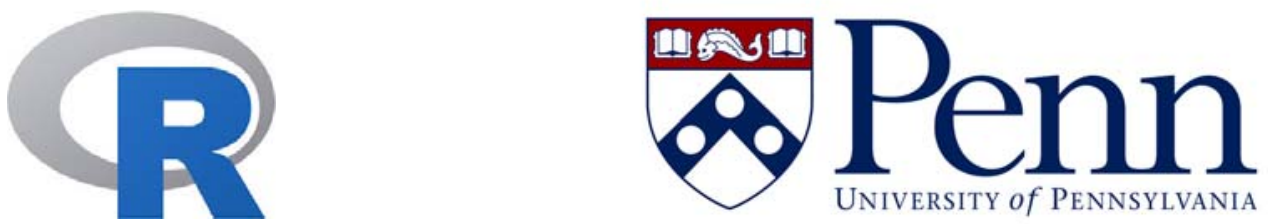

http://finzi.psych.upenn.edu/library/bayesvl/html/00Index.html

Open-source library server

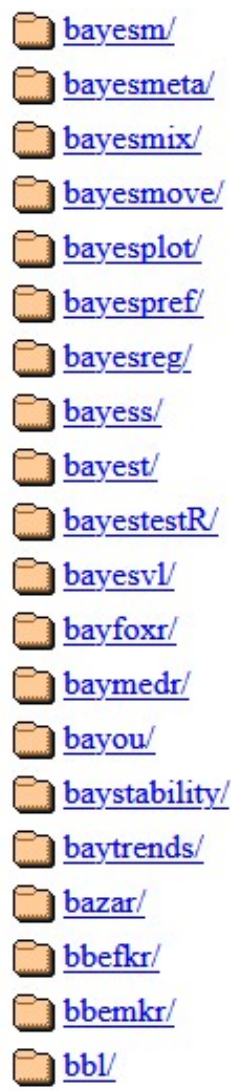

2020-01-21 16:03

2020-09-08 12:52

2016-12-20 16:08

2020-12-03 22:24

2020-12-03 19:47

2016-12-20 17:19

2019-06-25 06:08

2016-12-20 14:50

2020-09-08 12:13

2020-12-03 13:14

2020-12-03 22:25

2019-03-23 06:02

2020-09-08 15:55

2018-10-19 07:38

2018-04-02 22:24

2020-12-03 15:17

2019-03-22 10:41

2016-12-20 13:28

2016-12-20 13:28

2020-03-12 09:02

http://finzi.psych.upenn.edu/library/ 


\section{Visually Learning the Graphical structure of Bayesian Networks and Performing MCMC with 'Stan' [R logo]}

$[\mathrm{Up}][\mathrm{Top}]$
Documentation for package 'bayesvl' version 0.8 .5

- DESCRIPTION file. bayesvl-package

$\underline{\text { bayesvl }}$

bayesvl bnlearn

bayesvl bnlearn utilities

bayesvl graph utilities

bayesvl graphs

bayesvl plot utilities

bayesvl plots

bayesvl stan

bayesvl stan utilities

bayesvl-class

bvl addArc

bvl addArc-method

bvl addNode

bvl addNode-method

bvl bnBarchart

bvl bnBarchart-method

bvl bnBayes

bvl bnBayes-method

bvl bnPlot

bvl_bnPlot-method

bvl bnScore

bvl bnScore-method

bvl bnStrength

bvl_bnStrength-method

bvl_formula

bvl_model2Stan

bvl_modelFit

bvl_modelFit-method

bvl_plotAcfs

bvl_plotAreas

bvl_plotDensity

bvl plotDensity2d

bvl_plotDiag
BayesVL package for Bayesian statistical analyses in $\mathrm{R}$

BayesVL package for Bayesian statistical analyses in $\mathrm{R}$ bnlearn interface for bayesvl objects

bnlearn interface for bayesvl objects

Utilities to manipulate graphs

Utilities to manipulate graphs

Plot utilities for bayesvl objects

Plot utilities for bayesvl objects

Build RStan models from directed acyclic graph Build RStan models from directed acyclic graph

Class 'bayesvl': object class of bayesvl model

Utilities to manipulate graphs

Class 'bayesvl': object class of bayesvl model

Utilities to manipulate graphs

Class 'bayesvl': object class of bayesvl model

bnlearn interface for bayesvl objects

bnlearn interface for bayesvl objects

bnlearn interface for bayesvl objects

bnlearn interface for bayesvl objects

Plot utilities for bayesvl objects

bnlearn interface for bayesvl objects

bnlearn interface for bayesvl objects

bnlearn interface for bayesvl objects

bnlearn interface for bayesvl objects

bnlearn interface for bayesvl objects

Build RStan models from directed acyclic graph

Build RStan models from directed acyclic graph

Build RStan models from directed acyclic graph

Class 'bayesvl': object class of bayesvl model

Plot utilities for bayesvl objects

Plot utilities for bayesvl objects

Plot utilities for bayesvl objects

Plot utilities for bayesvl objects

Plot utilities for bayesvl objects 
bvl plotGelman

bvl_plotGelmans

bvl_plotIntervals

bvl_plotPairs

bvl plotParams

bvl_plotTest

bvl plotTrace

bvl_stanParams

bvl stanParams-method

bvl stanPriors

Legends345

show-method

summary-method
Plot utilities for bayesvl objects

Plot utilities for bayesvl objects

Plot utilities for bayesvl objects

Plot utilities for bayesvl objects

Plot utilities for bayesvl objects

Plot utilities for bayesvl objects

Plot utilities for bayesvl objects

Build RStan models from directed acyclic graph

Class 'bayesvl': object class of bayesvl model

Build RStan models from directed acyclic graph

Legends345 data

Class 'bayesvl': object class of bayesvl model

Class 'bayesvl': object class of bayesvl model 


\section{References}

La, V. P., \& Vuong, Q. H. (2019). bayesvl: Visually learning the graphical structure of Bayesian networks and performing MCMC with 'Stan'. The Comprehensive R Archive Network (CRAN). Available online: https://cran.r-project.org/package=bayesvl

Vuong, Q. H., \& La, V. P. (2019). BayesVL package for Bayesian statistical analyses in R. GitHub. Available online: https://github.com/sshpa/bayesvl

Vuong, Q. H., La, V. P., Nguyen, M. H., Ho, M. T., Ho, M. T., \& Mantello, P. (2020). Improving Bayesian statistics understanding in the age of Big Data with the bayesvl R package. Software Impacts, 4, 100016.

Vuong, Q. H., La, V. P., Nguyen, M. H., Ho, M. T., Tran, T., \& Ho, M. T. (2020). Bayesian analysis for social data: A step-by-step protocol and interpretation. MethodsX, 7, 100924. 